Klimaschutz in der Landwirtschaft

\section{Brachliegende Potenziale nutzen}

\author{
Die Bundesregierung möchte die angestrebte Reduktion von \\ Treibhausgasemissionen durch ein integriertes Energie- und Kli- \\ maprogramm erreichen. Die darin vorgesehenen Maßnahmen \\ zeigen, dass der Landwirtschaftssektor in der deutschen Klima- \\ schutzpolitik keine Rolle spielt. Zu Unrecht, bietet die Umstruk- \\ turierung der Landwirtschaft doch ein erhebliches Potenzial \\ zum Klimaschutz. Von Jesko Hirschfeld und Julika Weiß
}

n der Diskussion um Klimaschutzstrategien richtet sich bisher das Augenmerk von Politik und Öffentlichkeit hauptsächlich auf Energiewirtschaft, Industrie, Verkehrswesen und private Haushalte. Die Rolle der Landwirtschaft wird dagegen meist vernachlässigt, obwohl sie mit 133 Millionen Tonnen für über 13 Prozent der in Deutschland emittierten Treibhausgase (THG) verantwortlich ist. So ignoriert die Agrarpolitik auf deutscher und europäischer Ebene, abgesehen von der Förderung des Energiepflanzenanbaus, bisher die Klimawirkungen und die Klimaschutzpotenziale der Landwirtschaft weitestgehend.

Eine gerade erschienene Studie des Instituts für ökologische Wirtschaftsforschung (IÖW) lotet die erheblichen Potenziale für den Klimaschutz in der Landwirtschaft aus (1).

\section{Vorgehensweise und Methodik}

Ziel der Studie war es, die Klimawirkungen der landwirtschaftlichen Produktion in Deutschland im Bezug auf die wichtigsten Agrarprodukte abzuschätzen und dabei insbesondere zu untersuchen, inwiefern sich die konventionelle und die ökologische Landwirtschaft in ihren Klimawirkungen unterscheiden und wo die Vor- und Nachteile der einzelnen Verfahren liegen. Damit sollte herausgearbeitet werden, wo Potenziale zur Verbesserung des Klimaschutzes in der Landwirtschaft liegen.
Zur Identifikation der Klimawirksamkeit einzelner Produktionsverfahren wurde eine detaillierte Analyse vier zentraler landwirtschaftlicher Produkte, nämlich Weizen, Schweinefleisch, Milch und Rindfleisch, durchgeführt. Die Vorgehensweise bei der Bilanzierung der Klimawirkungen beruhte auf der normierten Methode der Ökobilanz, die der Abschätzung der mit einem Produkt verbundenen Umweltwirkungen dient. Die Klimabilanz beschränkt sich auf die Wirkungskategorie Treibhauseffekt.

Zur Berechnung der THG-Emissionen und als Datenbank wurde auf das Computerprogramm GEMIS zurückgegriffen, wobei für die betrachteten Verfahren eigene Datensätze auf der Grundlage aktueller Forschungsergebnisse generiert wurden. Die Klimabilanzierung erfolgte vergleichend für typische Verfahren der ökologischen und konventionellen Landwirtschaft. Zur Berücksichtigung dynamischer Innovationseffekte wurden neben Durchschnittsbetrieben jeweils auch Spitzenbetriebe, die sich hinsichtlich ihrer Leistungen innerhalb der oberen zehn Prozent ihrer jeweiligen Betriebstypen befinden, betrachtet.

Pro Kilogramm (kg) Weizen verursacht der ökologische Landbau weniger als halb so viel Treibhausgasemissionen wie der konventionelle Weizenanbau, nämlich 141 Gramm (g) Kohlenstoffdioxid $\left(\mathrm{CO}_{2}\right)$-Äquivalente pro $\mathrm{kg}$ Weizen gegenüber 365 g im konventionell-integrierten Anbau. Ähnliche Unterschiede bestehen auch beim Anbau anderer
Pflanzen. Insbesondere der Mineraldüngereinsatz im konventionellen Landbau erweist sich als Klimakiller. Bei der Herstellung des Mineraldüngers werden enorme Energiemengen eingesetzt, die mit hohen $\mathrm{CO}_{2}$-Emissionen verbunden sind. Außerdem sind die relativ hohen Stickstoffüberschüsse nach Ausbringung der Düngemittel auf die Ackerflächen verantwortlich für hohe Lachgasemissionen, die sehr klimarelevant sind.

\section{Klimawirkung unterschied- licher Produktionsverfahren}

Pro Kilogramm Schweinefleisch entstehen in der ökologischen Schweinemast knapp 40 Prozent weniger THG-Emissionen als in der konventionellen Schweinemast, nämlich $1,70 \mathrm{~kg} \mathrm{CO}$-Äquivalente pro kg Schweinefleisch gegenüber $2,79 \mathrm{~kg}$ in der konventionellen Schweinemast (siehe Abbildung 1). Die Vorteile der ökologischen Landwirtschaft beruhen vor allem auf den geringeren Emissionen beim Futtermittelanbau.

Bei der Produktion von Milch liegen die THG-Emissionen der ökologischen Milchviehhaltung um knapp neun Prozent unter denen der konventionellen Haltungsverfahren. Abbildung 2 zeigt das Verhältnis zwischen $630 \mathrm{~g} \mathrm{CO}_{2}$-Äquivalenten pro kg Milch gegenüber $700 \mathrm{~g}$ in der konventionellen Milcherzeugung. Eine Kuh aus ökologischer Haltung verursacht mit etwa $5.000 \mathrm{~kg} \mathrm{CO}$-Äquivalenten pro Jahr deutlich weniger als eine konventionelle Kuh mit etwa $7.000 \mathrm{~kg} \mathrm{CO}_{2}$-Äquivalenten. Dieser Klimavorteil der ökologischen Milcherzeugung wird jedoch durch die geringere Milchleistung von 5.500 bis 7.500 kg Milch pro Jahr im Gegensatz zu 7.500 bis $9.500 \mathrm{~kg}$ Milch pro Jahr weitgehend aufgezehrt. Milch aus ökologischer und konventioneller Landwirtschaft verursachen damit hohe THGEmissionen, weshalb auch die daraus produzierten Milchprodukte eine hohe Klimawirkung haben. So verursacht ein Kilogramm Käse allein durch die Erzeugung der dafür notwendigen Milchmenge zwischen sechs und 16 Kilogramm $\mathrm{CO}_{2}$-Äquivalente - mehr als fünfmal so viel wie ein Kilo Schweinefleisch aus ökologischer Erzeugung. 
Der Systemvergleich zwischen konventioneller und ökologischer Landwirtschaft kommt bei der Rindfleischerzeugung nicht zu eindeutigen Ergebnissen, da hier eine ganze Reihe verschiedener Verfahren nebeneinander existieren: Bullen- und Ochsenmast von Kälbern aus Milchvieh- oder Mutterkuhhaltung sowie die Schlachtung von Altkühen. Die hinsichtlich der produzierten Rindfleischmengen bedeutendsten Verfahren sind in der konventionellen Landwirtschaft die Bullenmast von Kälbern aus Milchviehhaltung, in der ökologischen Landwirtschaft die Ochsenmast von Kälbern aus Mutterkuhhaltung. Die konventionelle

Abbildung 1: Treibhausgasemissionen durch die Erzeugung von 1 kg Schweinefleisch (Schlachtgewicht; ab Hof)

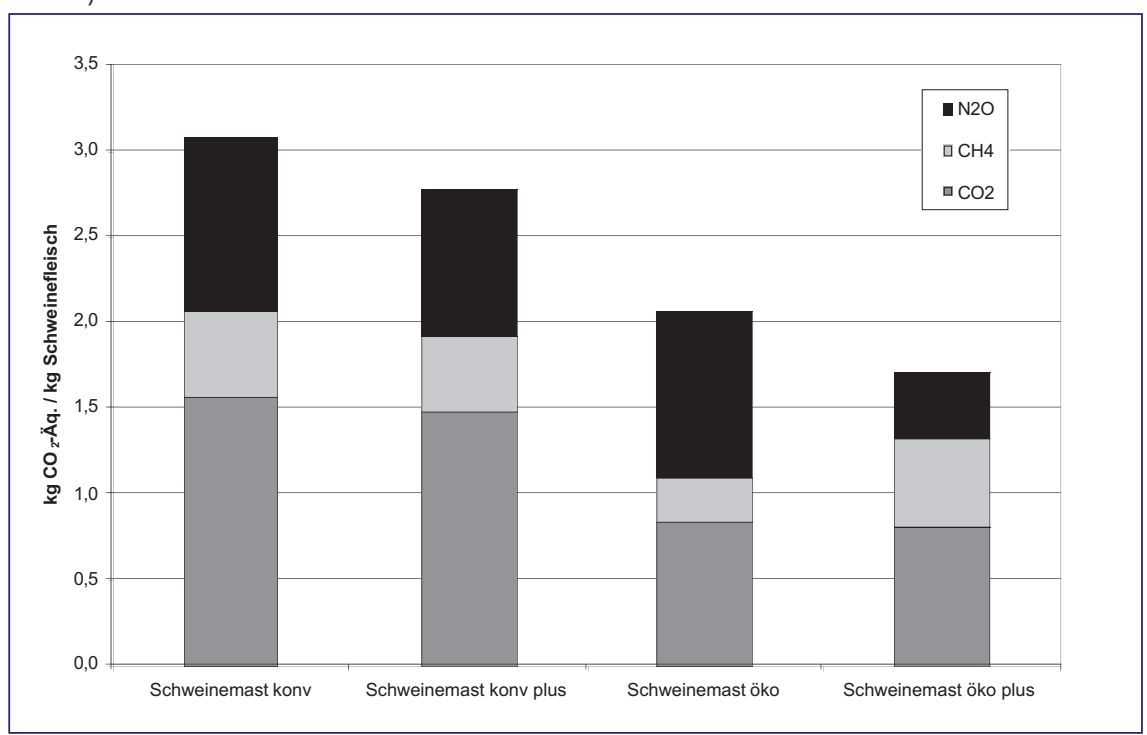

Treibhauspotenzial inkl. der Vorprodukte für Schweinefleisch von vier Modellbetrieben der Schweinemast: ein durchschnittlicher konventioneller Betrieb (konv.), ein technologisch führender, konventioneller Betrieb (konv. plus), ein durchschnittlicher Ökobetrieb (öko) und ein technologisch führender Ökobetrieb (öko plus) Quelle: : IÖW 2008

Abbildung 2: Treibhausgasemissionen aufgrund der Erzeugung von 1 kg Milch (Rohmilch ab Hof)

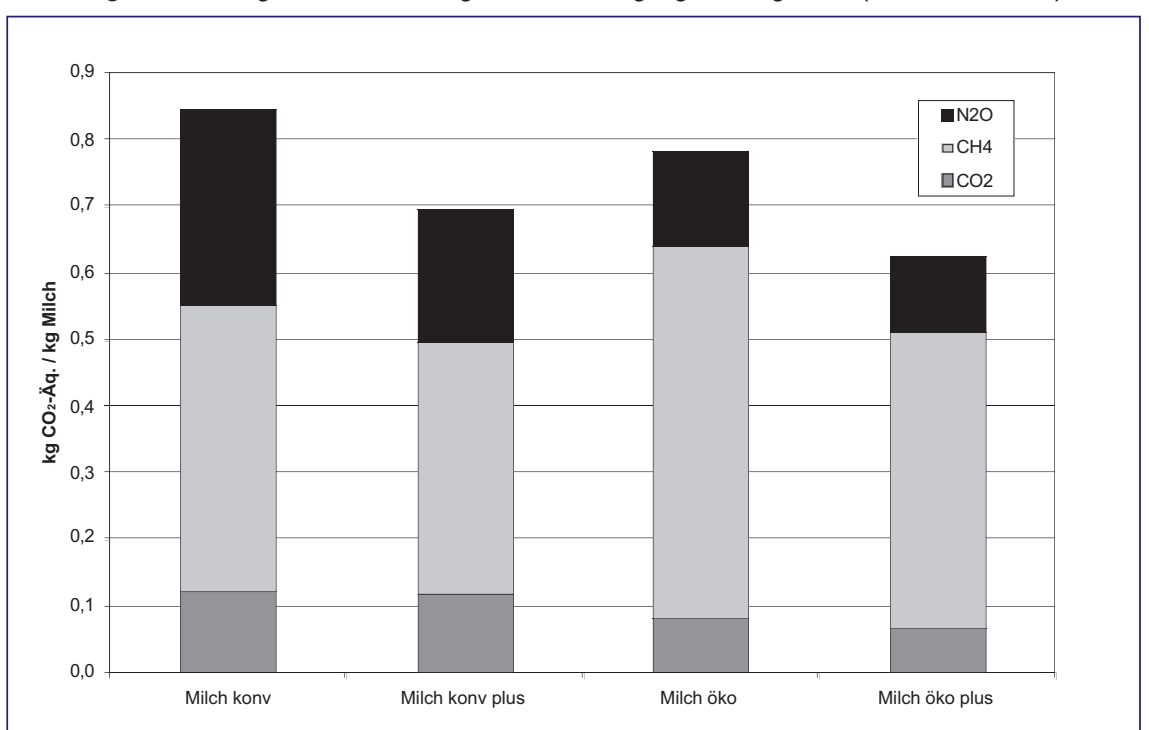

Treibhauspotenzial inkl. der Vorprodukte für Milch aus vier Modellbetrieben der Milchviehhaltung: ein durchschnittlicher konventioneller Betrieb (konv.), ein technologisch führender, konventioneller Betrieb (konv. plus), ein durchschnittlicher Ökobetrieb (öko) und ein technologisch führender Ökobetrieb (öko plus)

Quelle: IÖW 2008

Bullenmast von Kälbern aus der Milchviehhaltung verursacht mit 7,9 beziehungsweise $8,4 \mathrm{~kg} \mathrm{CO}$-Äquivalenten pro $\mathrm{kg}$ Rindfleisch nur rund halb so viel Treibhausgasemissionen wie die ökologische Ochsenmast von Kälbern aus der Mutterkuhhaltung mit 14,1 beziehungsweise $16,3 \mathrm{~kg} \mathrm{CO}$-Äquivalenten pro kg Rindfleisch. Die deutlich geringeren Emissionen des konventionellen Verfahrens resultieren zum einen daraus, dass die Kälber ein Nebenprodukt der Milcherzeugung darstellen. Zum anderen weisen die konventionellen Mastverfahren aber auch eine höhere Effektivität der Futterverwertung auf.

Der Einsatz von Biogasanlagen stellt eine Möglichkeit dar, die negativen Klimawirkungen der tierischen Produktionsverfahren zu verringern. Insbesondere bei den extensiven Verfahren der Rinderhaltung können mit bis zu 25 Prozent deutliche Einsparungen an THGEmissionen erzielt werden.

\section{Klimaschutzpotenziale der Landwirtschaft}

Auf der Grundlage der Ergebnisse zu einzelnen Produktionsbereichen wurde in der Studie eine überschlägige Abschätzung der Gesamteffekte, die durch eine klimafreundlichere Ausrichtung der Landwirtschaft für den Klimaschutz erreicht werden könnten, vorgenommen. Durch eine, in hohem Maße hypothetische, vollständige Umstellung auf die Verfahren der ökologischen Landwirtschaft könnten die Treibhausgasemissionen der deutschen Landwirtschaft um 20 Prozent beziehungsweise 27 Millionen Tonnen $\mathrm{CO}_{2}$-Äquivalente reduziert werden. Dabei sind es bis auf die Rindfleischerzeugung in der Bullenmast überwiegend die Verfahren des ökologischen Landbaus, die die Klimabilanz der Landwirtschaft gegenüber der gegenwärtigen Praxis verbessern könnten.

Die angegebenen Reduktionswerte könnten entweder durch eine nahezu vollständige Umstellung auf ökologischen Landbau oder aber durch eine durchgreifende Klimaoptimierung des konventionellen Landbaus erreicht werden. Diese 
würde unter anderem den nahezu vollständigen Verzicht auf den Einsatz von Mineraldünger beinhalten. Eine solche ressourcen- und klimaschutzoptimierte konventionelle Landwirtschaft würde sich der Wirtschaftsweise des ökologischen Landbaus teilweise annähern.

Bisher noch wenig ins Licht der Öffentlichkeit gerückt ist die Rolle der Bodennutzung für die Klimabilanz der Landwirtschaft. Auf nur fünf bis acht Prozent der landwirtschaftlichen Flächen entstehen 28 Prozent der Treibhausgasemissionen der deutschen Landwirtschaft, weil Moore entwässert und die kohlenstoffreichen Torfböden als Grünland und zum Ackerbau genutzt werden. Die organische Substanz wird über viele Jahrzehnte kontinuierlich abgebaut und setzt dabei enorme Mengen an Treibhausgasen frei. Zusammengenommen sind ackerbauliche Moornutzung und Grünlandentwässerung also verantwortlich für fast ein Drittel der landwirtschaftlichen Treibhausgasemissionen in Deutschland.

Durch die Wiedervernässung ackerbaulich genutzter Moorflächen, von gegenwärtig entwässertem Grünland sowie durch den Verzicht auf weiteren Grünlandumbruch wären entsprechend noch umfangreichere Klimaschutzziele zu erreichen, nämlich eine Reduktion von Treibhausgasemissionen von jährlich 37 Millionen Tonnen beziehungsweise 28 Prozent der landwirtschaftlichen THGEmissionen. Zusammen mit einer vollständigen Umstellung auf ökologischen Landbau beziehungsweise einer durchgreifenden Extensivierung der konventionellen Landwirtschaft ließe sich hypothetisch eine Reduktion um bis zu 68,6 Millionen Tonnen $\mathrm{CO}_{2}$-Äquivalente beziehungsweise die Hälfte der landwirtschaftlichen Treibhausgasemissionen erreichen. Dies entspräche gut einem Viertel der Treibhausgasemissionen, die die Bundesregierung bis zum Jahr 2020 zusätzlich zu den bisher erreichten Reduktionen vermeiden will.

Aus der hypothetischen Umstellung der gegenwärtigen Praxis auf die jeweils klimafreundlichsten Verfahren ergäbe sich allerdings ein Flächenmehrbedarf von rund 11,5 Millionen Hektar. Das sind etwa 68 Prozent der in Deutschland gegenwärtig genutzten landwirtschaftlichen Fläche. Da zusätzliche landwirtschaftliche Flächen in Deutschland nicht zur Verfügung stehen, müsste sich die Struktur der landwirtschaftlichen Erzeugung und des Nahrungsmittelkonsums verschieben. Kurz gesagt: Mehr Klimaschutz in der Landwirtschaft ist nur möglich, wenn der Anteil der Fleisch- und Milcherzeugung deutlich reduziert wird.

\section{Agrarpolitik und Klimaschutz}

Angesichts des hohen Beitrags der deutschen Landwirtschaft zu den gesamten THG-Emissionen und der großen Potenziale zu deren Verringerung ist es dringend geboten, dass die deutsche und europäische Agrarpolitik im Bezug auf ihre Klimawirkungen überprüft wird und die Bundesregierung den Bereich Landwirtschaft zukünftig in ihre Klimaschutzpolitik einbezieht. Dafür müssen nicht zuletzt alle agrarpolitischen Maßnahmen, die zu einer Aufrechterhaltung eines klimaschädlichen Intensitätsniveaus und Produktionsumfangs führen, auf den Prüfstand gestellt werden.

Die Berücksichtigung der Klimaeffekte der landwirtschaftlichen Produktion darf jedoch nicht den Blick verstellen auf Tierschutzaspekte und weitere Umweltwirkungen der Landwirtschaft. Eine einseitige Optimierung hinsichtlich der Klimawirkungen kann sonst wichtige andere Ziele des Umwelt- und Tierschutzes gefährden. So treten teilweise Nutzungskonkurrenzen zwischen landwirtschaftlichen Nutzflächen und naturschutzrelevanten Brach- und Waldflächen auf. In der Tierhaltung sind außerdem ethische Aspekte $\mathrm{zu}$ berücksichtigen, wenn beispielsweise die Vorteilhaftigkeit von verschiedenen Stallhaltungsformen bewertet wird. Viele Klimaschutzmaßnahmen in der Landwirtschaft zeichnen sich jedoch durch weitere positive Umwelteffekte aus, indem zum Beispiel durch die Wiedervernässung von Mooren ein Beitrag zum Schutz der Biodiversität geleistet wird oder die Reduktion des Düngemitteleinsatzes gleichzeitig zu einer Verringerung der Gewässerbelastung führt.
Auf der Grundlage der Ergebnisse der Studie ergeben sich folgende zentrale Ansatzpunkte für eine Klimaschutzstrategie im Bereich der Landwirtschaft:

- Wiedervernässung von entwässerten Moorflächen und Feuchtwiesen

I Reduktion des Einsatzes von Mineraldünger

I Klimaschutzoptimierung der Stallhaltung

- Steigerung der Erträge und Leistungen

I Nutzung von Gülle und Mist in Biogasanlagen

I Klimatechnische Optimierung der Rinderhaltung durch Kombination von Milch- und Fleischproduktion

- Verringerung des Imports von klimaschädlichen Futtermitteln

Um eine durchgreifende Reduzierung der Treibhausgasemissionen aus der Landwirtschaft zu erreichen, ist außerdem eine Veränderung der Struktur und der Menge der Fleischerzeugung und damit verbunden eine Veränderung des Fleischkonsums notwendig. So sind über 70 Prozent der Treibhausgasemissionen der deutschen Landwirtschaft auf die Fleischerzeugung zurückzuführen, wobei die Rindfleischerzeugung aus Klimaschutzperspektive sehr viel ungünstiger zu beurteilen ist als die Schweine- und Geflügelfleischerzeugung. Jede Reduzierung in diesem Bereich macht Flächen frei für pflanzliche Nahrungsmittel und verbessert die Klimabilanz der Landwirtschaft.

\section{Anmerkung}

(1) Download der Studie unter: www.ioew.de. Die Studie wurde im Auftrag von foodwatch e.V. erstellt und durch die Deutsche Wildtierstiftung, Karl-Ludwig Schweisfurth und die Klett-Stiftung

\section{AUTOREN + KONTAKT}

Dr. Jesko Hirschfeld ist wissenschaftlicher Mitarbeiter im Forschungsfeld Umweltökonomie und Umweltpolitik und Dr. Julika Weiß ist wissenschaftliche Mitarbeiterin im Forschungsfeld Nachhaltige Energiewirtschaft und Klimaschutz am Institut für ökologische Wirtschaftsforschung (IÖW).

Institut für ökologische Wirtschaftsforschung (IÖW), Potsdamer Str. 105, 10785 Berlin. Tel.: +49 30 884594-0, Fax.: +49308825493, E-Mail: Jesko.Hirschfeld@ioew.de, Julika.Weiss@ioew.de 
(c) 20I0 Authors; licensee IÖW and oekom verlag. This is an article distributed under the terms of the Creative Commons Attribution Non-Commercial No Derivates License (http://creativecommons.org/licenses/by-nc-nd/3.o/), which permits unrestricted use, distribution, and reproduction in any medium, provided the original work is properly cited. 